\title{
Rationale and design of ARTS: a randomized, double-blind study of BAY 94-8862 in patients with chronic heart failure and mild or moderate chronic kidney disease
}

\author{
Bertram Pitt ${ }^{1 *}$, Gerasimos Filippatos ${ }^{2}$, Mihai Gheorghiade ${ }^{3}$, Lars Kober ${ }^{4}$, \\ Henry Krum ${ }^{5}$, Piotr Ponikowski ${ }^{6}$, Christina Nowack ${ }^{7}$, Peter Kolkhof ${ }^{8}$, \\ So-Young Kim ${ }^{9}$, and Faiez Zannad ${ }^{10}$
}

${ }^{1}$ University of Michigan School of Medicine, Ann Arbor, MI 48109-0366, USA; ${ }^{2}$ Heart Failure Unit, Department of Cardiology, Attikon University Hospital, Athens, Greece; ${ }^{3}$ Center for Cardiovascular Innovation, Northwestern University Feinberg School of Medicine, Chicago, IL, USA; ${ }^{4}$ Heart Centre, Rigshospitalet, University of Copenhagen, Denmark; ${ }^{5}$ Department of Epidemiology and Preventive Medicine, Centre of Cardiovascular Research and Education in Therapeutics, Monash University, Melbourne, VIC, Australia; ${ }^{6}$ Medical University, Clinical Military Hospital, Wroclaw, Poland; ${ }^{7}$ Global Clinical Development, Bayer Pharma AG, Wuppertal, Germany; ${ }^{8}$ Cardiology Research, Department of Heart Diseases, Global Drug Discovery, Bayer HealthCare Pharmaceuticals, Wuppertal, Germany; ${ }^{9}$ Bayer Vital GmbH, Bayer HealthCare, Leverkusen, Germany; and ${ }^{10}$ INSERM, Centre for Clinical Investigation 9501 and Unit 961, Central University Hospital and the Department of Cardiology, Université de Lorraine, Nancy, France

Received 17 February 2012; revised 27 March 2012; accepted 30 March 2012

\begin{abstract}
Aims
BAY 94-8862 is a novel, non-steroidal, mineralocorticoid receptor antagonist with greater selectivity than spironolactone and stronger mineralocorticoid receptor binding affinity than eplerenone. The aims of the MinerAlocorticoid Receptor Antagonist Tolerability Study (ARTS; NCT01345656) are to evaluate the safety and tolerability of BAY 948862 in patients with heart failure associated with a reduced left ventricular ejection fraction (HFREF) and chronic kidney disease (CKD), and to examine the effects on biomarkers of cardiac and renal function.

Methods ARTS is a multicentre, randomized, double-blind, placebo-controlled, parallel-group study divided into two parts. In part A, oral BAY $94-8862$ [2.5, 5, or $10 \mathrm{mg}$ once daily (o.d.)] is compared with placebo in $\sim 60$ patients with HFREF and mild CKD. Outcome measures include serum potassium concentration, biomarkers of renal injury, estimated glomerular filtration rate (eGFR), and albuminuria. Part B compares BAY $94-8862$ (2.5, 5, or $10 \mathrm{mg}$ o.d., or $5 \mathrm{mg}$ twice daily), placebo, and open-label spironolactone $(25-50 \mathrm{mg}$ o.d.) in $\sim 360$ patients with HFREF and moderate CKD. Outcome measures include the change in serum potassium concentration with BAY 94-8862 vs. placebo (primary endpoint) and vs. spironolactone, safety and tolerability, biomarkers of cardiac and renal function or injury, eGFR, and albuminuria. BAY 94-8862 pharmacokinetics are also assessed.

Perspectives ARTS is the first phase II clinical trial of BAY 94-8862 and is expected to provide a wealth of information on BAY 948862 in patients with HFREF and CKD, including the optimal dose range for further studies.

Keywords

Aldosterone • Antagonist • Chronic kidney disease • Heart failure • Mineralocorticoid receptor
\end{abstract}

\section{Introduction}

In patients hospitalized for heart failure (HF), high levels of aldosterone are an important predictor of early re-hospitalization., ${ }^{1,2}$ The effects of aldosterone are mediated via activation of its specific nuclear hormone receptor, the mineralocorticoid receptor (MR).
Mineralocorticoid receptor antagonists (MRAs) have been found to be effective in reducing total mortality as well as frequency of hospitalizations for HF in: patients with chronic HF associated with a reduced left ventricular ejection fraction (HFREF); patients with HFREF following myocardial infarction; and patients with mild symptoms of HFREF. ${ }^{2-5}$ MRAs have also been shown to

\footnotetext{
* Corresponding author. Tel: + 1734936 5260, Fax: + 1734936 5256, Email: bpitt@med.umich.edu

Published on behalf of the European Society of Cardiology. All rights reserved. (C) The Author 2012. For permissions please email: journals.permissions@oup.com.
} 
lower blood pressure in patients with primary aldosteronism and individuals with essential hypertension, especially in those with resistant hypertension. ${ }^{6,7}$ Several studies have demonstrated that addition of an MRA to conventional therapy [an angiotensinconverting enzyme (ACE) inhibitor and/or an angiotensin receptor blocker (ARB)] in patients with chronic kidney disease (CKD) substantially reduces proteinuria compared with conventional therapy alone. $^{8-11}$ Reductions in markers of myocardial fibrosis have also been observed in patients receiving MRAs in combination with ACE inhibitors or ARBs. ${ }^{12}$

However, the use of MRAs in eligible patients remains suboptimal. ${ }^{13-15}$ The reluctance of some clinicians to use the available MRAs, spironolactone and eplerenone, in HFREF despite their proven effects on mortality is largely attributable to their fear of inducing hyperkalaemia, especially in patients with concomitant diabetes mellitus and/or CKD. The use of spironolactone (a firstgeneration MRA) is also limited by significant progestogenic and antiandrogenic activity owing to its structural similarity to progesterone; ${ }^{16,17}$ this activity has been associated with the occurrence of breast pain in both sexes, gynaecomastia and impotence in males, and menstrual irregularities in females. ${ }^{4,18}$ Eplerenone (a secondgeneration MRA) is more specific for the MR than spironolactone, but the results of two head-to-head comparator studies in patients with hypertension suggest that eplerenone may have reduced antihypertensive efficacy compared with spironolactone. ${ }^{19,20}$

These limitations of spironolactone and eplerenone have stimulated further research, leading to the discovery of new non-steroidal MRAs that are more selective for the MR than spironolactone and have greater affinity for the MR than eplerenone. ${ }^{21,22}$ To reduce the risk of hyperkalaemia, a next-generation MRA is needed that has more pronounced cardiac activity in comparison with the available steroidal MRAs, so that improvements in cardiac function can be achieved without deleterious changes to sodium-potassium homeostasis in the kidney. Such a compound could result in MRAs being used in a larger number of patients with $\mathrm{HF}$ and $\mathrm{CKD}$, as well as in other indications such as acute HF syndromes, ${ }^{23}$ thereby potentially reducing cardiovascular mortality, hospitalizations for HF, and healthcare resource use.
BAY 94-8862 is a novel, non-steroidal, next-generation MRA. ${ }^{22}$ In vitro studies indicate that BAY $94-8862$ has superior selectivity compared with spironolactone and improved affinity for the MR compared with eplerenone (Table 1). The safety and tolerability of different oral doses of BAY 94-8862 have been assessed and confirmed in healthy volunteers (data on file). We report the design of the MinerAlocorticoid Receptor Antagonist Tolerability Study (ARTS), the first phase II clinical trial of BAY 94-8862. The study has been initiated to evaluate the safety and tolerability of different oral doses of BAY 94-8862 in patients with HFREF and CKD, and to examine its effect on biomarkers of cardiac and renal function or injury.

\section{Methods}

\section{Study design}

ARTS (ClinicalTrials.gov identifier: NCT01345656) is a multicentre, randomized, double-blind, placebo-controlled, parallel-group study divided into two parts: part A was conducted in patients with HFREF and mild CKD [estimated glomerular filtration rate (eGFR) 60 to $<90 \mathrm{~mL} / \mathrm{min} / 1.73 \mathrm{~m}^{2}$ ), and part $B$ is currently being conducted in patients with HFREF and moderate CKD (eGFR $30-60 \mathrm{~mL} / \mathrm{min} /$ $1.73 \mathrm{~m}^{2}$ ). Part $B$ has an open-label active comparator (spironolactone) group in addition to the placebo control group (Figure 1).

\section{Objectives}

The objective of part A was to investigate the safety, tolerability, and renal effects of oral BAY 94-8862 compared with placebo. The effects of BAY 94-8862 on serum potassium concentration, biomarkers of renal injury, eGFR [calculated using the Modification of Diet in Renal Disease (MDRD) formula], ${ }^{24}$ and albuminuria were assessed, as well as the pharmacokinetics of BAY 94-8862 and its metabolites in plasma after multiple oral doses.

The primary objective of part $B$ is to investigate the change in serum potassium concentration after treatment with oral BAY $94-8862$ compared with placebo. Secondary objectives are to compare the change in serum potassium concentration in the BAY 94-8862 and spironolactone groups, to assess safety and tolerability of BAY 94-8862, and to examine the effects of BAY 94-8862 on biomarkers of cardiac and renal function or injury, eGFR (MDRD), and albuminuria. In addition,

Table I In vitro potency and selectivity of spironolactone, eplerenone, and BAY 94-8862 in functional cell-based steroid hormone receptor transactivation assays ${ }^{25,26}$

\begin{tabular}{|c|c|c|c|}
\hline & Spironolactone & Eplerenone & BAY 94-8862 \\
\hline Mineralocorticoid receptor $\mathrm{IC}_{50}(\mathrm{nM})$ & 24.2 & 990 & 17.8 \\
\hline Glucocorticoid receptor $\mathrm{IC}_{50}(\mathrm{nM})$ & 2410 & $\geq 21980$ & $\geq 10000$ \\
\hline Androgen receptor $\mathrm{IC}_{50}(\mathrm{nM})$ & 77.1 & $\geq 21240$ & $\geq 10000$ \\
\hline Progesterone receptor $\mathrm{IC}_{50} / \mathrm{EC}_{50}^{\mathrm{a}}(\mathrm{nM})$ & $740^{\mathrm{a}}$ & $\geq 31210$ & $\geq 10000$ \\
\hline Oestrogen receptor $\alpha \mathrm{IC}_{50}(\mathrm{nM})$ & 5970 & $\geq 30000$ & $\geq 10000$ \\
\hline Oestrogen receptor $\beta$ IC 50 (nM) & 4940 & $\geq 30000$ & $\geq 10000$ \\
\hline
\end{tabular}

Values were determined using Chinese hamster ovary (CHO)-K1 cells stably expressing the ligand-binding domains of the oxo-steroid receptors. Data were obtained from at least four independent experiments performed in duplicate, except for the oestrogen receptor data, which were obtained from two independent experiments. The respective steroid hormone cell lines and assay procedures have been described previously. ${ }^{25}$

$E C_{50}$, half-maximal effective concentration; $I C_{50}$, half-maximal inhibitory concentration.

${ }^{a}$ Spironolactone is an agonist of the progesterone receptor and the respective $\mathrm{EC}_{50}$ value is therefore given. 


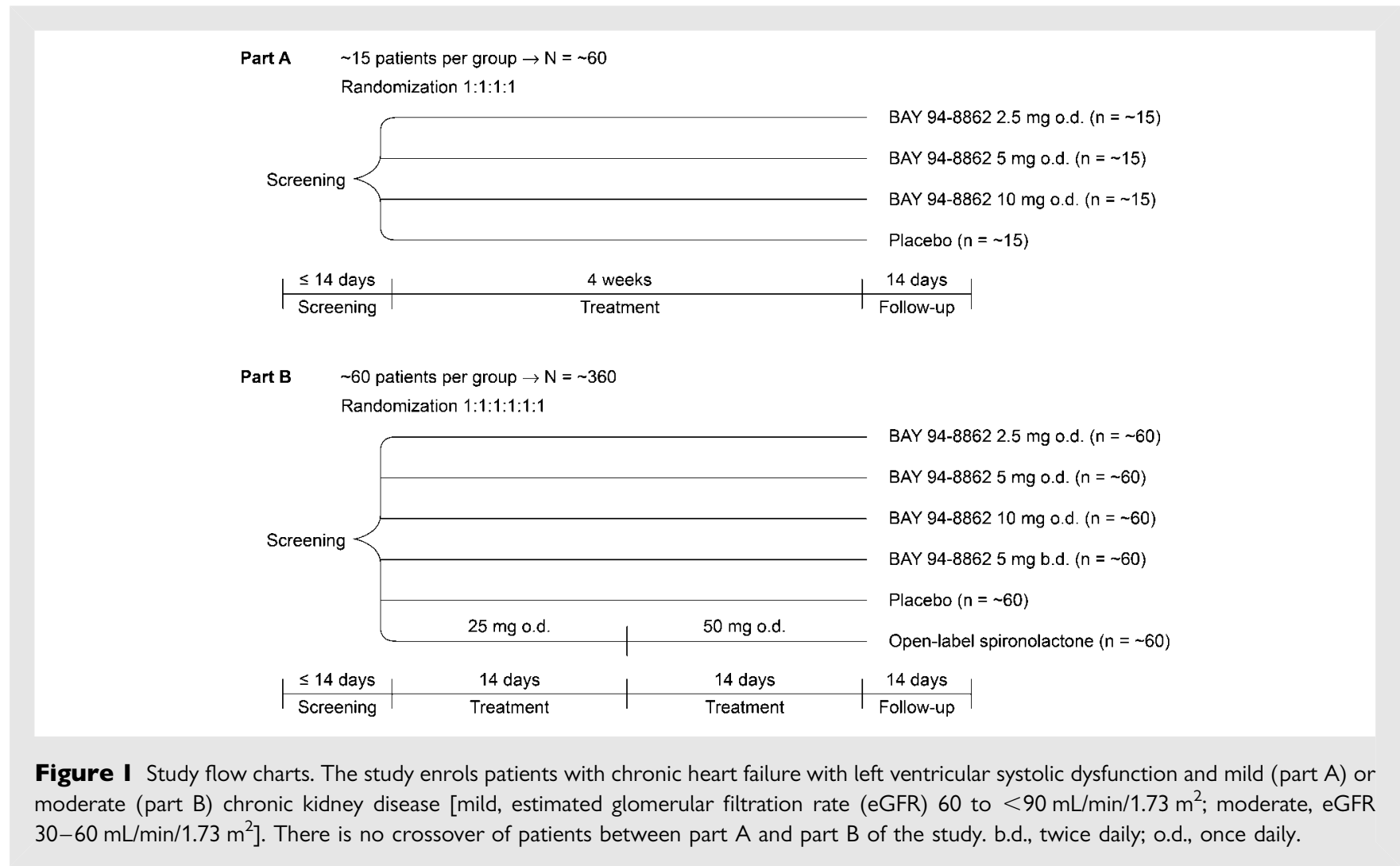

the pharmacokinetics of BAY 94-8862 in plasma after multiple oral doses will be assessed.

\section{Patients}

Adult males and females without childbearing potential with a clinical diagnosis of HFREF [New York Heart Association (NYHA) class IIIII], treated with evidence-based therapy for HFREF, and who meet all of the inclusion criteria and none of the exclusion criteria (Table 2) will be eligible for enrolment in the study.

Patients must be withdrawn from the study if: they decline to participate further; continuation of the study would be harmful to the patient's well-being in the opinion of the investigator; it is specifically requested by the sponsor; overall study drug intake is $<80 \%$ or $>120 \%$ of the prescribed dose; any adverse event (AE) occurs that is not acceptable in the opinion of the investigator and/or patient; or the patient develops worsening cardiac function that requires hospitalization and initiation of intravenous drug treatment. In addition, if serum potassium is between 5.5 and $6.0 \mathrm{mmol} / \mathrm{L}$, a second blood sample must be taken as soon as possible, at the latest on the next day. If serum potassium is still $>5.5 \mathrm{mmol} / \mathrm{L}$ in the second sample, the study drug must be discontinued permanently. If serum potassium is $>6.0 \mathrm{mmol} / \mathrm{L}$ in any blood sample, the study drug must be discontinued permanently without any further laboratory assessment.

\section{Study medication}

BAY $94-8862$ is administered as oral, immediate-release tablets. In both parts of the study, eligible patients are randomized in the 14 days following a screening visit, and receive the study drug for 4 weeks. In part $A$, eligible patients were randomized 1:1:1:1 to receive BAY $94-8862$ at 2.5 , 5 , or $10 \mathrm{mg}$ once daily (o.d.) or placebo. In part B, eligible patients are randomized 1:1:1:1:1:1 to receive BAY $94-8862$ at 2.5, 5, or $10 \mathrm{mg}$ o.d., BAY $94-8862$ at $5 \mathrm{mg}$ twice daily (b.d.), placebo, or open-label spironolactone (initial dose $25 \mathrm{mg}$ o.d. up-titrated to $50 \mathrm{mg}$ o.d. on day $15 \pm 1$ if the serum potassium concentration is $\leq 4.8 \mathrm{mmol} / \mathrm{L}$ ). There is no crossover of patients between part $A$ and part $B$ of the study.

Treatment with evidence-based therapy for HFREF is a requirement for inclusion in the study (Table 2) and may be continued during the course of the study, apart from the exceptions noted below. Concomitant medications not allowed during the study (from initiation of study drug treatment until the follow-up visit has been completed) include: any aldosterone antagonist or renin inhibitor; use of an ACE inhibitor with an ARB; potassium-sparing agents such as potassium-sparing diuretics; high-dose acetylsalicylic acid (>500 mg/day); daily treatment with non-steroidal anti-inflammatory agents; potent cytochrome P450 (CYP) isoenzyme 3A4 inhibitors or inducers; and strong CYP2C8 inhibitors such as gemfibrozil.

\section{Investigations}

Participating patients are assessed at the screening visit, at baseline/day 1 (start of study drug administration), day $4 \pm 1$, day $8 \pm 1$, and weekly thereafter until the end of the study. A follow-up visit will be scheduled 14 days after the last intake of study medication. Patients who discontinue the study prematurely are also assessed as soon as possible after discontinuation. In part $\mathrm{B}$, patients receiving spironolactone at the up-titrated dose of $50 \mathrm{mg}$ o.d. are assessed at an additional visit on day $18 \pm 1$.

Details of the assessments made at each visit are shown in Table 3. Briefly, patients undergo a physical examination at the screening visit, and demographic data and the medical history are also recorded. Continuous assessment of AEs starts immediately 


\section{Table 2 Inclusion and exclusion criteria}

Inclusion criteria

- Written informed consent signed before any study-specific procedure

- Men aged $\geq 18$ years or post-menopausal women aged $\geq 55$ years or women aged $\geq 18$ years not of childbearing potential based on surgical treatment such as bilateral tubal ligation, bilateral ovarectomy, or hysterectomy

- Clinical diagnosis of chronic heart failure, either ischaemic or non-ischaemic, NYHA class II-III for at least 3 months ${ }^{\mathrm{a}}$

- Treated with evidenced-based therapy for chronic heart failure with LVSD (e.g. beta-blockers and ACE inhibitors or ARBs, as well as diuretics, unless contraindicated or not tolerated)

- Stable patients (NYHA class II-III) naïve to aldosterone antagonist therapy and stable, low-risk patients (NYHA class II) with ongoing aldosterone antagonist therapy that, in the opinion of the investigator, may be safely withdrawn for the duration of the study (maximum 10 weeks)

- A record of LVEF $\leq 40 \%$ (measured by any modality) in the patient's medical history without intervening revascularization, cardiac surgery, or implantation of biventricular pacemaker in the meantime; for those patients with cardiac intervention, LVEF must be re-assessed and an LVEF $\leq 40 \%$ re-confirmed after the cardiac intervention

- Known kidney damage for $\geq 3$ months, as defined by structural or functional abnormalities of the kidney, and:

- part A: eGFR 60 to $<90 \mathrm{~mL} / \mathrm{min} / 1.73 \mathrm{~m}^{2}$ (MDRD) at screening visit

- part B: eGFR 30-60 mL/min/1.73 $\mathrm{m}^{2}$ (MDRD) at screening visit

- Serum potassium $\leq 4.8 \mathrm{mmol} / \mathrm{L}$ at screening visit

- Systolic blood pressure $\geq 90 \mathrm{mmHg}$ without signs or symptoms of hypotension at screening visit

- Ability to understand and follow study-related instructions

Exclusion criteria

- Women of childbearing potential

- Known hypersensitivity to the study drug (active substance or excipients) or spironolactone and respective excipients (part B only)

- Patients with anuria, acute renal failure, or Addison's disease

- Worsening heart failure requiring hospitalization and treatment with intravenous diuretics in the 30 days before the screening visit for patients (NYHA class II-III) naïve to aldosterone antagonist therapy or in the 6 months before the screening visit for patients (NYHA class II) who, immediately before study entry, are receiving aldosterone antagonist therapy

- Acute coronary syndrome or unstable coronary artery disease in the 30 days before randomization

- Valvular heart disease requiring surgical intervention during the course of the study

- Evidence of increased ventricular vulnerability (e.g. survived ventricular fibrillation, sustained ventricular tachycardia, or firing of implantable cardioverter-defibrillator in the 30 days before randomization) requiring any intervention during the course of the study

- History of hospitalization for hyperkalaemia or acute renal failure induced by previous aldosterone antagonist treatment

- History of or clinically significant evidence of any severe disease other than chronic heart failure that would preclude participation in the study

- Patients with clinically relevant hepatic dysfunction at screening visit indicated by one of the following: total bilirubin concentration more than twice the ULN and alanine aminotransferase levels more than three times the ULN or hepatic insufficiency classified as Child-Pugh B or C

- Use of any renin inhibitor or aldosterone antagonist in the 30 days before randomization

- Concomitant therapy with potassium-sparing agents, high-dose acetylsalicylic acid ( $>500 \mathrm{mg} /$ day), or continuous treatment with non-steroidal anti-inflammatory agents

- Concomitant therapy with potent CYP isoenzyme 3A4 inhibitors or inducers (to be stopped $\geq 7$ days before randomization) or strong CYP2C8 inhibitors (to be stopped $\geq 48 \mathrm{~h}$ before randomization) such as gemfibrozil (investigators will be provided with a list of concomitant medications considered potent CYP3A 4 inhibitors or inducers or strong CYP2C8 inhibitors)

- Ongoing drug or alcohol abuse

- Concomitant regular liquorice consumption

- Uncontrolled hypertension at the screening visit requiring additional antihypertensive treatment during the course of the study

- Clinically relevant findings from the physical examination that may influence absorption, distribution, metabolism, elimination, or effects of the study drugs, or jeopardize the patient's safety during the study

- Poorly controlled diabetes mellitus with glycated haemoglobin $>8.5 \%$ at screening visit

- Participation in another clinical study or treatment with another investigational product in the 30 days before randomization

- Any other condition or therapy that will make the patient unsuitable for this study and will not allow participation for the full planned study period

- Previous assignment to treatment during this study

ACE, angiotensin-converting enzyme; ARB, angiotensin receptor blocker; CYP, cytochrome P450; eGFR, estimated glomerular filtration rate; LVEF, left ventricular ejection fraction; LVSD, left ventricular systolic dysfunction; MDRD, Modification of Diet in Renal Disease; MRA, mineralocorticoid receptor antagonist; NYHA, New York Heart Association; ULN, upper limit of the normal range.

aMRA-naive patients can be considered for participation in this study independently of their NYHA class (II-III) at the screening visit. Interruption of ongoing MRA treatment should be considered only in patients with NYHA class II, not hospitalized for worsening heart failure in the 6 months before the screening visit, and only if the investigator considers the patient's estimated individual risk of a cardiovascular event to be acceptable.

after signing the informed consent form until the follow-up visit or the premature discontinuation visit (if applicable). For each $A E$, the investigator records the maximum intensity (mild, moderate, or severe), seriousness, relationship to the study drug, any actions taken, and the final outcome. At intervals during the course of the study, concomitant medications are noted and vital signs (heart rate and blood pressure) and 12-lead electrocardiograms (ECGs) are assessed after resting for at least $10 \mathrm{~min}$. 
Table 3 Schedule of assessment

\begin{tabular}{|c|c|c|c|c|c|c|c|c|c|c|}
\hline Visit & Screen & 1 (Baseline) & 2 & 3 & 4 & $5^{a}$ & 6 & 7 & PD & 8 (Follow-up) \\
\hline Day & $\leq-14$ & 1 & $4 \pm 1$ & $8 \pm 1$ & $15 \pm 1$ & $18 \pm 1$ & $22 \pm 2$ & $29 \pm 2$ & & $43 \pm 5$ \\
\hline Informed consent & $x$ & & & & & & & & & \\
\hline Assess eligibility & $x$ & $x$ & & & & & & & & \\
\hline Demographic data & $x$ & & & & & & & & & \\
\hline Medical history & $x$ & & & & & & & & & \\
\hline Physical examination & $x$ & & & & & & & $x$ & $x$ & $x$ \\
\hline 12-lead ECG & $x$ & $x$ & & $x$ & $x$ & & $x$ & $x$ & $x$ & $x$ \\
\hline Vital signs & $x$ & $x$ & & $x$ & $x$ & & $x$ & $x$ & $x$ & $x$ \\
\hline PK blood sampling ${ }^{\mathrm{b}}$ & & $x$ & $x$ & $x$ & $x$ & & $x$ & $x$ & & \\
\hline Haematology & $x$ & $x$ & & $x$ & $x$ & & $x$ & $x$ & $x$ & $x$ \\
\hline Clinical chemistry & $x$ & $x$ & $x$ & $x$ & $x$ & $x$ & $x$ & $x$ & $x$ & $x$ \\
\hline Urinalysis & $x$ & $x$ & & & $x$ & & & $x$ & $x$ & $x$ \\
\hline Blood and urine samples for biomarkers & & $x$ & & & $x$ & & & $x$ & $x$ & $x$ \\
\hline Adverse events & $x$ & $x$ & $x$ & $x$ & $x$ & $x$ & $x$ & $x$ & $x$ & $x$ \\
\hline Concomitant medications & $x$ & $x$ & $x$ & $x$ & $x$ & $x$ & $x$ & $x$ & $x$ & $x$ \\
\hline
\end{tabular}

ECG, electrocardiogram; PD, premature discontinuation; PK, pharmacokinetics.

${ }^{a}$ Visit 5 is performed only in part B and only in patients who were up-titrated to spironolactone $50 \mathrm{mg}$ once daily at visit 4 (day 15).

${ }^{b}$ Not applicable to patients on open-label treatment with spironolactone.

Blood samples are taken to measure levels of thyroid-stimulating hormone, free triiodothyronine, and free thyroxine, as well as glycated haemoglobin $\left(\mathrm{HbA}_{1 \mathrm{c}}\right)$, serum creatinine (from which the eGFR is calculated), and cystatin C. Biomarkers indicating renal injury [kidney injury molecule (KIM)-1 and neutrophil gelatinase-associated lipocalin (NGAL)] are measured in urine. In part $B$, levels of biomarkers reflecting cardiac function [brain natriuretic peptide (BNP), N-terminal proBNP, ultrasensitive troponin I, asymmetric dimethylarginine (ADMA), osteopontin, galectin-3, and aldosterone] are measured in blood. Urine samples are taken to measure the urinary creatinine:albumin ratio. Standard clinical chemistry tests are also performed.

In addition, blood samples for pharmacokinetic analysis are taken at pre-specified time points. In each case, the time of study drug intake and the sampling time will be recorded. The plasma concentrations of BAY 94-8862 are determined using a sparse sampling approach in all participants.

\section{Study organization}

Data from part A were reviewed for safety and tolerability by an independent data monitoring committee (DMC) in August 2011. The safety and tolerability of different doses in patients with HFREF and mild CKD were confirmed by the DMC. Part B was started in patients with HFREF and moderate CKD at the beginning of September 2011.

\section{Statistical considerations}

\section{Statistical methodology}

There will be four analysis sets: the safety set (all patients who have taken at least one dose of study drug); the full analysis set (all individuals from the safety set with baseline and at least one post-baseline serum potassium values); the per protocol set (all patients from the full analysis set of part B with valid serum potassium data at visit 6 or 7 and no major protocol deviations); and the pharmacokinetic analysis set (all patients who complete the study without major protocol deviations). In part A, all variables will be analysed descriptively in the safety set. In part B, the primary analysis will be performed in the full analysis set, with a supportive analysis in the per protocol set.

The primary safety variable in part B will be the change in mean serum potassium values from baseline at visits 6 and 7. Five different dose-response models [linear, quadratic, exponential, logistic, or maximum effect $\left.\left(E_{\max }\right)\right]$ will be fitted to the serum potassium data from the placebo and BAY 94-8862 groups, and the best model will be selected based on the Akaike and Bayesian information criteria.

Based on the selected dose-response model, the $95 \%$ confidence interval $(\mathrm{Cl})$ will be constructed, and the BAY 94-8862 dose range that increases serum potassium concentration more than placebo but less than spironolactone will be identified. An exploratory statistical comparison will be performed between the BAY 94-8862 dose groups that are within this range and the spironolactone group.

In addition, changes in biomarkers, eGFR (MDRD formula), albuminuria, serum potassium and magnesium concentrations, blood pressure, and heart rate will be assessed using analysis of covariance. The numbers of patients with serum potassium concentrations $>5.5 \mathrm{mmol} / \mathrm{L}$ and $6.0 \mathrm{mmol} / \mathrm{L}$ will be analysed by Fisher's exact test. The analyses for the above secondary variables will be performed in the full analysis set and per protocol analysis set; all other safety analyses will be performed in the safety set. Based on a sparse sampling approach performed at multiple visits, the pharmacokinetics of BAY 94-8862 will be analysed using population-pharmacokinetic methods. Safety laboratory parameters and ECG data will be analysed descriptively only.

\section{Sample size}

A normally distributed data set with a common standard deviation of serum potassium of $0.5 \mathrm{mmol} / \mathrm{L}$ (based on literature research) and mean values that have a linear relationship with the dose was simulated for a sample size of 60 patients in the placebo group and each BAY 94-8862 treatment group. A linear line was fitted and the $95 \% \mathrm{Cl}$ was constructed. For the given sample size, the width of the one-sided $95 \% \mathrm{Cl}$ was $\sim 0.08 \mathrm{mmol} / \mathrm{L}$. 
For the exploratory comparison of the BAY 94-8862 dose groups with the spironolactone group, assuming a common standard deviation of $0.5 \mathrm{mmol} / \mathrm{L}$ for change in serum potassium concentration and 60 patients per group, each comparison will have $80 \%$ power to detect a difference between the BAY 94-8862 dose group and the spironolactone group.

\section{Ethical considerations}

The procedures set out in this protocol pertaining to the conduct, evaluation, and documentation of this study are designed to ensure that the sponsor and investigator abide by Good Clinical Practice guidelines and follow the guiding principles detailed in the Declaration of Helsinki. The study is carried out in keeping with applicable local law(s) and regulation(s). Documented approval from appropriate independent ethics committee(s) or institutional review board(s) was obtained for all participating centres/countries before the start of the study.

\section{Discussion}

In order to avoid or at least diminish the untoward side effect of hyperkalaemia associated with currently available MRAs, drugs with a more pronounced cardiac or vascular activity, at least in comparison with the available steroidal MRAs, are desirable so that improvements in cardiac function can be achieved without deleterious changes to sodium-potassium homeostasis in the kidney. Screening for novel non-steroidal MRAs led to the identification of dihydropyridine-based compounds such as BR-4628, which occupies the MR ligand-binding cavity differently from classical steroidal MRAs. ${ }^{25}$ A medicinal chemistry optimization programme culminated in the identification of BAY $94-8862,{ }^{26}$ which is at least as potent as spironolactone, even more selective than eplerenone (Table 1), and has no residual L-type calcium channel blocking activity, in contrast to related dihydropyridine-based MRAs. ${ }^{26-28}$ The physicochemical properties that drive binding to plasma proteins, tissue permeability, and distribution of this novel compound may lead to a more favourable balance of cardiac antiremodelling effects vs. renal (electrolyte) effects compared with approved MRAs, ultimately leading to an improved clinical safety profile with a reduced risk of hyperkalaemia.

ARTS has been designed to address several key questions related to the potential efficacy and safety of BAY 94-8862. The inclusion of both a placebo group and a positive control group treated with spironolactone $(25-50 \mathrm{mg}$ o.d.) should allow insight into the relative effects of BAY 94-8862 on renal function and the incidence of hyperkalaemia in patients with HFREF and moderate CKD treated with standard therapy (including an ACE inhibitor or ARB, a beta-blocker, and diuretics), many of whom are expected to have concomitant diabetes mellitus. The open-label nature of the spironolactone treatment will have to be considered when interpreting the results, because investigators may reduce potassium more in the spironolactone group than in the other four groups.

It has been suggested that MRAs may have beneficial effects on the kidneys in patients with CKD. A systematic review of clinical studies in patients with CKD found that addition of MRAs to standard therapy led to a reduction in proteinuria compared with placebo, although a significant improvement in eGFR was not observed. ${ }^{11}$ There has not, however, been a large trial powered to evaluate renal and cardiovascular outcomes in patients with CKD, due to the fear of inducing serious hyperkalaemia with the presently available MRAs. A retrospective analysis of almost 25000 veterans in the USA found that the incidence of hyperkalaemia (serum potassium $>5.5 \mathrm{mmol} / \mathrm{L}$ ) was more than three times higher in patients with CKD than in those without CKD, regardless of renin-angiotensin-aldosterone system inhibitor status, and that there was an increased mortality associated with a serum potassium concentration $>5.5 \mathrm{mmol} / \mathrm{L}$ within $24 \mathrm{~h}$ of its occurrence. ${ }^{29}$ To ensure the safety of BAY $94-8862$ and its proposed dosing in ARTS, patients with HFREF and mild CKD (part A) were evaluated and monitored by the DMC before patients with HFREF and moderate CKD were entered into the study (part B).

In ARTS, renal effects of BAY $94-8862$ are monitored by several parameters including serum creatinine, eGFR, the urinary albumin:creatinine ratio, and exploratory biomarkers of renal injury, such as NGAL and KIM-1. Effects on cardiac function and injury will also be assessed by monitoring the concentrations of various biomarkers including BNP, NT-proBNP, and ultrasensitive troponin I. The duration of the treatment period (4 weeks) and the follow-up after termination of study medication (14 days) should allow an assessment of the optimal effectiveness of BAY 94-8862 on the MR.

In addition to assessing safety and effects on markers of end-organ function and injury, ARTS will provide information on the pharmacokinetic properties of BAY 94-8862 and its optimal dosing in patients with HFREF and CKD. The study has been designed to determine doses of BAY 94-8862 that cause a significantly lower increase in serum potassium and incidence of hyperkalaemia than spironolactone (25-50 mg o.d.), while having significantly greater efficacy than placebo and at least similar efficacy to spironolactone, as assessed by levels of BNP or NT-proBNP, ultrasensitive troponin I, ADMA, galectin-3, and osteopontin. The relative potency at the MR may be assessed indirectly by measuring the changes in aldosterone levels. In patients with HFREF and CKD, these biomarkers should provide initial insight into the relative effects of BAY 94-8862 on collagen formation, ventricular remodelling, and nitric oxide availability, all of which have been associated with cardiovascular mortality in patients with HFREF and/or CKD.

\section{Conclusion}

Heart failure remains a highly prevalent disease with high mortality despite current treatment. The presently available steroidal MRAs spironolactone and eplerenone have been shown to improve outcomes, but adverse effects and the need for frequent monitoring of serum potassium and renal function throughout treatment have limited their uptake in routine clinical practice. Several potential new indications for MRAs are yet to be systematically investigated. BAY 94-8862 is a novel non-steroidal MRA that could potentially combine high potency (similar to spironolactone) with high selectivity (greater than eplerenone), improved end-organ protective activity, and improved safety compared with previous MRAs, based on differences in physicochemical properties. ARTS-a multicentre, randomized, double-blind, placebo-controlled study-is the first clinical trial of BAY 94-8862 in patients and is expected 
to provide a wealth of information on BAY 94-8862 in individuals with HFREF and CKD. Should the hypothesis prove correct and BAY 94-8862 is demonstrated to have improved safety at a given efficacy than presently available steroidal MRAs in these high-risk patients with HFREF and moderate CKD, this would open the way for larger clinical outcome studies in this patient group and possibly other indications, potentially leading to reductions in cardiovascular mortality, hospitalizations for HF, and healthcare resource use.

\section{Funding}

Bayer HealthCare AG. Dr Claire Mulligan and Dr Charlotte Cookson of Oxford PharmaGenesis ${ }^{\text {TM }}$ Ltd provided medical writing support funded by Bayer HealthCare AG.

Conflicts of interest: B.P. has received consulting fees from Bayer, Pfizer, Merck, Novartis, Takeda, AstraZeneca, Lilly, GE Healthcare, Relypsa, BG Medicine, Amorcyte, Cytopherx, and Aurasense, stock options from Relypsa, BG Medicine, and Aurasense, and grants from Novartis, Medtronic, and Forest Laboratories. G.F. has received consulting fees from Bayer. M.G. has been a consultant for Abbott Laboratories, Astellas, AstraZeneca, Bayer HealthCare AG, Corthera, Cytokinetics, Debiopharm S.A., Errekappa Terapeutici, GlaxoSmithKline, Ikaria, Johnson \& Johnson, Medtronic, Merck, Novartis Pharma AG, Otsuka Pharmaceuticals, Palatin Technologies, PeriCor Therapeutics, Protein Design Laboratories, Sanofi-Aventis, Sigma-Tau, Solvay Pharmaceuticals, Takeda Pharmaceutical, and Trevena Therapeutics. L.K. has received consulting fees from Bayer HealthCare AG, Novartis Pharma AG, and Sanofi-Aventis. H.K. has received consulting fees from Bayer. P.P. has received consulting fees from Bayer and speaker honoraria from Pfizer. C.N. is an employee of Bayer Pharma AG. P.K. is an employee of Bayer HealthCare Pharmaceuticals. S.-Y.K. is an employee of Bayer Vital GmbH. F.Z. has received speaker/consultant honoraria from Alere, AstraZeneca, BG Medicine, Boston Scientific, Novartis, Pfizer, Resmed, Servier, and Takeda.

ARTS investigators: Austria: F. Fruhwald, H. Drexel, A. Podczeck-Schweighofer, J. Altenberger, P. Siostrzonek; Belgium: A-C. Pouleur, W. Droogné, W. Smolders, M. De Ceuninck; Czech Republic: J. Spinar, E. Nemecek, F. Malek, T. Belza; Germany: E. Vester, B. R. Winkelmann, F. Baer, W. Sehnert, J. Beermann, F. Richard, U. Schulze, S. Anker, A. Al-Zoebi, H. Hohensee; Denmark: L. Køber, C. Torp-Pedersen, H. Nielsen, S. Lind Rasmussen, K. Skagen, K. Egstrup, L. Videbæk, T. Nielsen; Finland: V.-P. Harjola, H. Ukkonen, K. Nyman; Israel: A. Marmor, A. Katz, T. B. Gal, S. Goland, A. Shotan, G. Keren, Y. Turgeman; Norway: S. Ørn, A. Westheim; Poland: P. Ponikowski; E. Mirek-Bryniarska, J. Niegowska, M. Ogorek, W. Krysiak; Sweden: A. Ohlsson, C.-J. Lindholm, I. Torstensson, R. Zlatewa, M. Rosenqvist.

\section{Appendix}

Steering Committee: Bertram Pitt (Chair; Ann Arbor, MI, USA); Faiez Zannad (Co-Chair; Nancy, France); Gerasimos Filippatos (Athens, Greece); Mihai Gheorghiade (Chicago, IL, USA); Lars Kober (Copenhagen, Denmark); Henry Krum (Melbourne, Victoria, Australia); Piotr Ponikowski (Wroclaw, Poland).

Data Monitoring Committee: Aldo Maggioni (Firenze, Italy); Marco Metra (Brescia, Italy); Kenneth Dickstein (Stavanger, Norway); Luis Ruilope (Madrid, Spain); Tim Collier (London, UK).
Study coordinators: Catherine Salt, Cat Taylor (Bayer plc, Newbury, UK); Seija Karinkanta, (Bayer Oy, Espoo, Finland); Veronique Smets (Bayer SA-NV, Diegem, Machelen, Belgium).

Statisticians: Nina Kimmeskamp-Kirschbaum (Bayer HealthCare Pharmaceuticals, Wuppertal, Germany); Daniel Haverstock (Bayer HealthCare Pharmaceuticals Inc., Montville, CT, USA).

Data managers: Jeannette Gerstner (Bayer HealthCare Pharmaceuticals, Berlin, Germany); Michael Hersch (Bayer Vital GmbH, Leverkusen, Germany).

\section{References}

1. Gheorghiade M, Pang PS, Ambrosy AP, Lan G, Schmidt P, Filippatos G, Konstam M, Swedberg K, Cook T, Traver B, Maggioni A, Burnett J, Grinfeld L, Udelson J, Zannad F. A comprehensive, longitudinal description of the in-hospital and post-discharge clinical, laboratory, and neurohormonal course of patients with heart failure who die or are re-hospitalized within 90 days: analysis from the EVEREST trial. Heart Fail Rev 2012;17:485-509.

2. McMurray JJ. CONSENSUS to EMPHASIS: the overwhelming evidence which makes blockade of the renin-angiotensin-aldosterone system the cornerstone of therapy for systolic heart failure. Eur J Heart Fail 2011;13:929-936.

3. Pitt B, Remme W, Zannad F, Neaton J, Martinez F, Roniker B, Bittman R, Hurley S, Kleiman J, Gatlin M. Eplerenone, a selective aldosterone blocker, in patients with left ventricular dysfunction after myocardial infarction. N Engl J Med 2003;348: 1309-1321.

4. Pitt B, Zannad F, Remme WJ, Cody R, Castaigne A, Perez A, Palensky J, Wittes J. The effect of spironolactone on morbidity and mortality in patients with severe heart failure. Randomized Aldactone Evaluation Study Investigators. N Engl J Med 1999;341:709-717.

5. Zannad F, McMurray JJ, Krum H, van Veldhuisen DJ, Swedberg K, Shi H, Vincent J, Pocock SJ, Pitt B. Eplerenone in patients with systolic heart failure and mild symptoms. N Engl J Med 2011;364:11-21.

6. Funder JW, Carey RM, Fardella C, Gomez-Sanchez CE, Mantero F, Stowasser M, Young WF Jr, Montori VM. Case detection, diagnosis, and treatment of patients with primary aldosteronism: an endocrine society clinical practice guideline. J Clin Endocrinol Metab 2008;93:3266-3281.

7. Epstein M, Calhoun DA. Aldosterone blockers (mineralocorticoid receptor antagonism) and potassium-sparing diuretics. J Clin Hypertens (Greenwich) 2011;13: 644-648.

8. Bianchi S, Bigazzi R, Campese VM. Long-term effects of spironolactone on proteinuria and kidney function in patients with chronic kidney disease. Kidney Int 2006;70:2116-2123.

9. Bomback AS, Kshirsagar AV, Amamoo MA, Klemmer PJ. Change in proteinuria after adding aldosterone blockers to ACE inhibitors or angiotensin receptor blockers in CKD: a systematic review. Am J Kidney Dis 2008;51:199-211.

10. Jain G, Campbell RC, Warnock DG. Mineralocorticoid receptor blockers and chronic kidney disease. Clin J Am Soc Nephrol 2009;4:1685-1691.

11. Navaneethan SD, Nigwekar SU, Sehgal AR, Strippoli GF. Aldosterone antagonists for preventing the progression of chronic kidney disease. Cochrane Database Syst $\operatorname{Rev} 2009(3)$ :CD007004.

12. Kosmala W, Przewlocka-Kosmala M, Szczepanik-Osadnik H, Mysiak A, O'Moore-Sullivan T, Marwick TH. A randomized study of the beneficial effects of aldosterone antagonism on LV function, structure, and fibrosis markers in metabolic syndrome. JACC Cardiovasc Imaging 2011;4:1239-1249.

13. Albert NM, Yancy CW, Liang L, Zhao $X$, Hernandez AF, Peterson ED, Cannon CP, Fonarow GC. Use of aldosterone antagonists in heart failure. JAMA 2009:302:1658-1665.

14. Fonarow GC, Yancy CW, Albert NM, Curtis AB, Stough WG, Gheorghiade M, Heywood JT, McBride ML, Mehra MR, O'Connor CM, Reynolds D, Walsh MN. Heart failure care in the outpatient cardiology practice setting: findings from IMPROVE HF. Circ Heart Fail 2008;1:98-106.

15. Maggioni AP, Dahlstrom U, Filippatos G, Chioncel O, Leiro MC, Drozdz J, Fruhwald F, Gullestad L, Logeart D, Metra M, Parissis J, Persson H, Ponikowski P, Rauchhaus M, Voors A, Nielsen OW, Zannad F, Tavazzi L. EURObservational Research Programme: the Heart Failure Pilot Survey (ESC-HF Pilot). Eur J Heart Fail 2010;12:1076-1084.

16. Corvol P, Michaud A, Menard J, Freifeld M, Mahoudeau J. Antiandrogenic effect of spirolactones: mechanism of action. Endocrinology 1975;97:52-58.

17. Weber KT. Aldosterone in congestive heart failure. N Engl J Med 2001;345: 1689-1697.

18. Jackson EK. Diuretics. In: Hardman JG, Limbird LE (eds). Goodman \& Gilman's: The Pharmacological Basis of Therapeutics. 10th ed. New York: McGraw-Hill; 2001. p780-781. 
19. Weinberger MH, Roniker B, Krause SL, Weiss RJ. Eplerenone, a selective aldosterone blocker, in mild-to-moderate hypertension. Am J Hypertens 2002;15: 709-716.

20. Parthasarathy HK, Menard J, White WB, Young WF Jr, Williams GH, Williams B, Ruilope LM, Mclnnes GT, Connell JM, MacDonald TM. A double-blind, randomized study comparing the antihypertensive effect of eplerenone and spironolactone in patients with hypertension and evidence of primary aldosteronism. J Hypertens 2011;29:980-990.

21. Funder JW. Mineralocorticoid-receptor blockade, hypertension and heart failure. Nat Clin Pract Endocrinol Metab 2005;1:4-5.

22. Kolkhof $\mathrm{P}$, Borden SA. Molecular pharmacology of the mineralocorticoid receptor: prospects for novel therapeutics. Mol Cell Endocrinol 2012;350:310-317.

23. Albaghdadi M, Gheorghiade M, Pitt B. Mineralocorticoid receptor antagonism: therapeutic potential in acute heart failure syndromes. Eur Heart J 2011;32: 2626-2633.

24. Levey AS, Bosch JP, Lewis JB, Greene T, Rogers N, Roth D. A more accurate method to estimate glomerular filtration rate from serum creatinine: a new prediction equation. Modification of Diet in Renal Disease Study Group. Ann Intern Med 1999;130:461-170
25. Fagart J, Hillisch A, Huyet J, Barfacker L, Fay M, Pleiss U, Pook E, Schafer S, Rafestin-Oblin ME, Kolkhof P. A new mode of mineralocorticoid receptor antagonism by a potent and selective nonsteroidal molecule. J Biol Chem 2010;285: 29932-29940.

26. Bärfacker L, Kuhl A, Hillisch A, Grosser R, Figueroa-Pérez $S$, Heckroth $H$, Nitsche A, Ergüden JK, Gielen-Haertwig H, Schlemmer KH, Mittendorf J, Paulsen H, Platzek J, Kolkhof P. Discovery of BAY 94-8862: a non-steroidal antagonist of the mineralocorticoid receptor for the treatment of cardiorenal diseases. ChemMedChem 2012; in press.

27. Arhancet GB, Woodard SS, Dietz JD, Garland DJ, Wagner GM, lyanar K, Collins JT, Blinn JR, Numann RE, Hu X, Huang HC. Stereochemical requirements for the mineralocorticoid receptor antagonist activity of dihydropyridines. J Med Chem 2010;53:4300-4304.

28. Dietz JD, Du S, Bolten CW, Payne MA, Xia C, Blinn JR, Funder JW, Hu X. A number of marketed dihydropyridine calcium channel blockers have mineralocorticoid receptor antagonist activity. Hypertension 2008;51:742-748.

29. Einhorn LM, Zhan M, Hsu VD, Walker LD, Moen MF, Seliger SL, Weir MR, Fink JC. The frequency of hyperkalemia and its significance in chronic kidney disease. Arch Intern Med 2009;169:1156-1162. 